Paediatr. Paedolog. 2021 · 56:59-66 https://doi.org/10.1007/s00608-021-00870-0 Angenommen: 15. Februar 2021 Online publiziert: 23. März 2021

(๑) Der/die Autor(en) 2021

Bei der spinalen Muskelatrophie (SMA) handelt es sich um eine seltene neuromuskuläre Erkrankung, bedingt durch einen genetischen Defekt, der zu einem fortschreitenden Untergang von Motoneuronen im Vorderhorn des Rückenmarks führt. Klinisch zeigt sich eine chronisch progrediente, nicht reversible Muskelschwäche bzw. -atrophie und führt unbehandelt bei der infantilen Form SMA 1 meist innerhalb der ersten 24 Lebensmonate zum Tod.

In den letzten Monaten hat dieses Krankheitsbild sehr viel Aufmerksamkeit auf sich gezogen, da nun erstmals medikamentöse Therapiemöglichkeiten zur Verfügung stehen und von vielen Seiten eine Aufnahme in das Neugeborenen-Screening verlangt wurde. Um diese Forderung nachvollziehen zu können, muss man die genetische Grundlage der Erkrankung und die Wirkungsweise der Medikamente verstehen.

\section{Genetik - Grundlage für neue Therapieansätze}

Der genetische Defekt liegt auf dem langen Arm des Chromosoms 5, weshalb diese Erkrankung auch 5q-assoziierte SMA genannt wird. Der Erbgang ist autosomal rezessiv. In der Normalbevölkerung beträgt die Carrier-Frequenz etwa 1 von 35 bis 40, das bedeutet, dass etwa 1 von 6000 bis 10.000 Neugeborenen betroffen ist.

Die Wahrscheinlichkeit, dass ein betroffenes Paar (beide Elternteile sind Träger des Gendefekts) ein an SMA erkranktes Kind bekommt, beträgt somit $25 \%$ [1-3].

\title{
Astrid Eisenkölbl
}

Universitätsklinik für Kinder- und Jugendheilkunde, Kepler Universitätsklinikum Linz, Linz, Österreich

\section{Neue Therapiemöglichkeiten der spinalen Muskelatrophie}

\section{Brauchen wir ein Neugeborenen-Screening?}

Im Jahr 1995 wurde das Gen, das für die SMA verantwortlich ist, identifiziert und Survival-motor-neuron-Gen 1 genannt (SMN1) [4]. Meist liegt eine homozygote Deletion des Exons 7 und/oder 8 auf diesem Gen vor, bei etwa $5 \%$ der Patienten findet man eine Deletion auf einem Allel und eine Punktmutation auf dem zweiten Allel.

Im menschlichen Genom existiert eine nahezu identische Kopie des SMN1Gens, nämlich das SMN2-Gen. Dieses unterscheidet sich vom SMN1-Gen nur durch ein einziges Nukleotid (Austausch $\mathrm{C}$ zu T auf Exon 7), aufgrund dessen verändert sich das Spleißen und Exon 7 wird bei der Transkription ausgelassen, sodass größtenteils ein trunkiertes, biologisch nicht wirksames Protein entsteht $[5,6]$.

Das SMN2-Gen liegt bei allen Patienten in unterschiedlicher Kopienanzahl vor. Da nur etwa $10 \%$ des Proteins, das von jedem einzelnen SMN2-Gen codiert wird, funktionstüchtig ist, spielt die Kopienanzahl eine große Rolle bezüglich dem Schweregrad der Erkrankung.

Die Übergänge sind dabei jedoch fließend. Ein Großteil der Patienten mit SMA 1 hat meist nur eine oder zwei SMN2-Kopien, während Patienten mit SMA 2 oder SMA 3 meist drei oder vier SMN2-Kopien vorweisen ([1, 7]; - Abb. 1 und 2).

\section{Klinisches Erscheinungs- bild - immer wieder eine Herausforderung}

Durch den Verlust der Motoneuronen kommt es zu einer proximal betonten symmetrischen Schwäche der Extremitäten und der Rumpfmuskulatur.

- Der häufigste Typ ist SMA 1, oder Werdnig-Hoffmann genannt, mit Erkrankungsbeginn innerhalb der ersten 12 Lebensmonate. Klinisch fallen die Säuglinge durch eine ausgeprägte muskuläre Hypotonie, eine schlechte Kopfkontrolle und durch eine rasche respiratorische Insuffizienz auf. Faszikulationen der Zunge sind ebenfalls ein typisches klinisches Zeichen für diese Erkrankung.

- Patienten mit SMA 2 weisen eine proximale Muskelschwäche auf und entwickeln häufig Gelenkskontrakturen bzw. Skoliosen. Der Erkrankungsbeginn liegt vor dem 18. Lebensmonat.

- Kinder mit SMA 3 zeigen eine beinbetonte Muskelschwäche und weisen eine normale Lebenserwartung auf. Hier liegt der Erkrankungsbeginn zwischen 18 Monaten und 30 Jahren.

- Sehr selten werden Neugeborene bereits mit massiven Kontrakturen und respiratorischer Insuffizienz geboren. Dabei handelt es sich dann um die schwerste Verlaufsform SMA 0 und die Lebenserwartung beträgt nur wenige Wochen.

- Ebenso selten, aber deutlich milder im Verlauf, gibt es einen Krankheitsbeginn im Erwachsenenalter (SMA 4; [1-3, 5, 7-9]; • Tab. 1)

Durch die Verfügbarkeit neuer Medikamente hat sich der Verlauf der Erkrankung verändert. Die Übergänge zwischen den einzelnen Typen waren immer schon fließend und werden sich nun noch weiter vermischen. In Zukunft wird man 


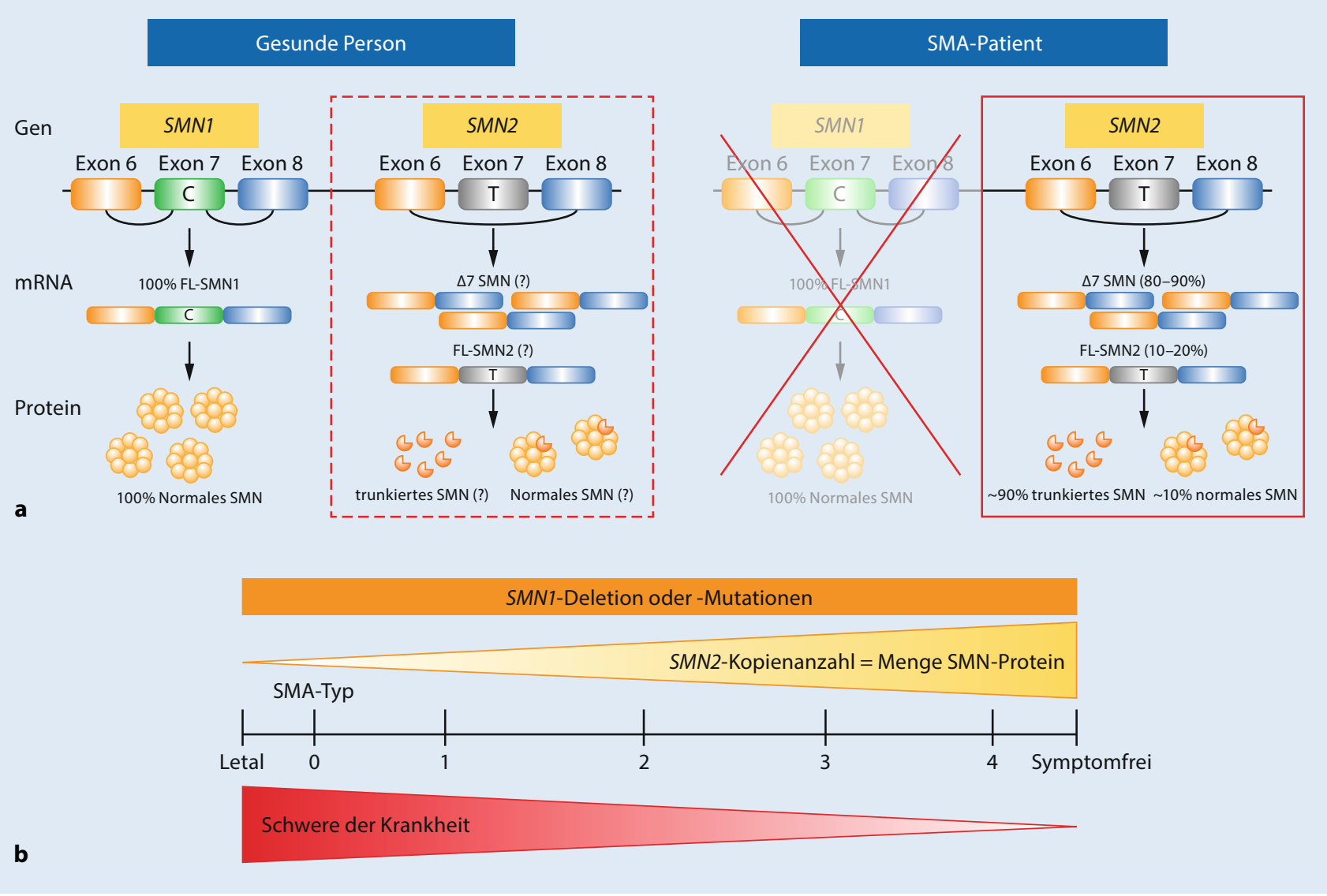

Abb. 1 a Bei gesunden Personen kann durch das SMN1-Gen 100\% funktionstüchtiges SMN-Protein gebildet werden, das SMN2-Gen produziert aufgrund des Fehlers auf Exon 7 nur eingeschränkt funktionstüchtiges Protein. Beim SMA-Patienten fehlt SMN1-Protein komplett, SMN 2 kann den Ausfall nur geringfügig kompensieren. $\mathbf{b}$ Es besteht eine Korrelation zwischen dem Krankheitsverlauf und der Anzahl der SMN2-Kopien [18]

von einem breiten Spektrum von leicht zu schwer und von Verläufen mit frühem oder spätem Beginn sprechen.

Allen Studien gemein ist, dass sich klar gezeigt hat, dass ein früher Therapiebeginn die besten Ergebnisse ermöglicht. Kinder mit schwerer Muskelschwäche und langer Krankheitsdauer profitieren wahrscheinlich nur sehr wenig von diesen Therapien, da sich Motoneurone, die zugrunde gegangen sind, nicht mehr erholen können.

Kinder, die kurz nach der Geburt oder zumindest vor Symptombeginn behandelt wurden, zeigten häufig die besten Ergebnisse in den motorischen Scores und erreichten annähernd die motorischen Meilensteine wie gesunde Kinder im gleichen Alter.

\section{Therapiemöglichkeiten - Fortschritte der letzten Jahre}

In den letzten Jahren hat sich in den Therapiemöglichkeiten der SMA sehr viel verändert. Erstmals stehen uns zur Behandlung der SMA Medikamente mit verschiedenen Therapieansätzen (• Abb. 3) zur Verfügung. Die vielversprechendsten befassen sich mit der Erhöhung der Produktion von SMNProtein, entweder über einen SplicingModifier, der auf dem SMN2-Gen ansetzt, oder über einen Ersatz des defekten SMN1-Gens.

\section{Nusinersen (Spinraza ${ }^{\circledR}$ )}

Nusinersen ist seit 2017 für die Behandlung von allen 5q-assoziierten SMA zugelassen. Bei Nusinersen handelt es sich um ein Antisense-Oligonukleotid, das das mRNA-Splicing verändert und so den Einschluss des Exons 7 auf dem SMN2-Gen fördert, womit mehr funktionierendes SMN-Protein zur Verfügung steht.

Das Medikament kann die Blut-HirnSchranke nicht überwinden und muss daher intrathekal verabreicht werden [10, 11].

Die Zulassung von Nusinersen basiert auf den Daten der beiden Zulassungsstudien ENDEAR, die Säuglinge unter 7 Monate mit SMA 1 einschloss, und CHERISH, die Kinder mit SMA 2 und 3 untersuchte. Beide Studien boten bei der Zwischenanalyse derart überzeugende Ergebnisse, dass sie aus ethischen Gründen vorzeitig gestoppt wurden, um den unbehandelten Kindern aus den Placebogruppen das Medikament nicht vorzuenthalten $[11,12]$.

In der ENDEAR-Studie haben $51 \%$ der Kinder motorische Meilensteine erreicht, im Vergleich zu $0 \%$ der Kinder in 
der Placebogruppe. Weiterhin kam es zu einer 47\%igen Reduktion von Tod bzw. permanenter mechanischer Beatmung (Tracheostoma oder Beatmung $\geq 16 \mathrm{~h}$ pro Tag für $\geq 21$ Tage; $[11,12])$.

In der CHERISH-Studie zeigten $57 \%$ in der Nusinersen-Gruppe vs. $26 \%$ in der Kontrollgruppe einer Verbesserung in den motorischen Scores. Dabei zeigten jene mit der kürzeren Krankheitsdauer die besseren Ergebnisse.

Aktuell werden präsymptomatische Kinder in der NURTURE-Studie untersucht: 25 Kinder wurden in diese Studie eingeschlossen und noch vor der 6. Lebenswoche mit Nusinersen behandelt. Nach einem medianen Follow-up von 2,9 Jahren sind alle Kinder am Leben, alle können ohne Unterstützung sitzen, $92 \%$ können mit Hilfe gehen, 88 \% sogar frei [12].

\section{„Small molecules"}

Bei den „small molecules“ handelt es sich um oral verfügbare Splicing-Modifier. Wie auch bei Nusinersen setzen diese Medikamente am SMN2-Gen an. Sie erhöhen den Einbau von Exon 7 und somit die Produktion von funktionstüchtigem SMN-Protein. Diese „small molecules“ können die Blut-Hirn-Schranke überwinden und die SMN-Proteinproduktion sowohl im ZNS als auch in der Peripherie bewirken.

Eines davon ist Risdiplam, das im August 2020 von der FDA für die Behandlung der SMA ab dem Alter von 2 Monaten zugelassen wurde. Der Antrag auf Zulassung in Europa liegt der EMA vor und mit einer Zulassung wird 2021 gerechnet [12].

\section{Onasemnogene abeparvovec -} xioi (Zolgensma ${ }^{\circledR}$ )

Seit Mai 2020 ist nun auch Zolgensma ${ }^{\circledR}$ in Europa zur Behandlung von Kindern mit SMA mit zwei oder drei SMN2Kopien und maximal $21 \mathrm{~kg}$ Körpergewicht zugelassen. Dabei handelt es sich um eine Genersatztherapie. Mittels eines Virusvektors (adenoassoziierter Virus 9) wird ein funktionierendes SMN1Gen in die Zellen eingebracht, das dort ein funktionierendes SMN-Protein bil-
Hier steht eine Anzeige.

㳕 Springer 
den kann. Zur Zulassung hat eine Studie geführt, in der 15 Kinder mit SMA 1 unter 9 Lebensmonaten eingeschlossen wurden. Drei der Kinder erhielten eine Dosis von AVXS-101 von $6,7 \times 10^{13} \mathrm{vg} / \mathrm{kg}$, 12 der Kinder erhielten eine höhere Dosis mit $2,0 \times 10^{14} \mathrm{vg} / \mathrm{kg}$. Verabreicht wurde das Medikament mittels einer einmaligen intravenösen Infusion. Diese wurde von allen Kindern gut toleriert, es kam lediglich zu erhöhten Transaminasen, die eine gewisse Reaktion der Leber auf den Adenovirus implementieren, die durch eine Kortisontherapie gut behandelt werden konnte. Von den 12 Patienten, die die höhere Dosis erhielten, erlernten 11 das freie Sitzen und sogar 2 das freie Gehen $[12,13]$.

Auch dieses Medikament wurde bei präsymptomatischen Patienten untersucht. Dabei wurden Patienten mit zwei oder drei SMN2-Kopien eingeschlossen, die am ehesten eine SMA 1 oder 2 entwickeln würden. In der Zwischenanalyse zeigte sich, dass 6 von 10 Kindern das freie Sitzen zum gleichen Zeitpunkt wie ein gesundes Kind erreichten und die anderen etwas später.

\section{Neugeborenen-Screening}

Das österreichische NeugeborenenScreening wurde bereits in den 1960erJahren etabliert, ist für die Familien kostenlos und umfasst verschiedene Stoffwechselerkrankungen sowie Hormonstörungen (kongenitale Hypothyreose, adrenogenitales Syndrom) und die zystische Fibrose. Damit können diese Erkrankungen frühzeitig erkannt und behandelt werden.

Da nun Therapiemöglichkeiten für die SMA zur Verfügung stehen und alle Studien darauf hinweisen, dass ein früher, möglichst präsymptomatischer Therapiebeginn vorteilhaft ist, wird derzeit versucht, die SMA in das NeugeborenenScreening aufzunehmen.

In den USA ist dies bereits in etwa 30 Bundesstaaten geglückt, in Deutschland fand seit Januar 2018 ein Pilotprojekt in den beiden Bundesländern Bayern und Nordrhein-Westfalen statt.

In diesen beiden Bundesländern wurden zwischen Januar 2018 und November 2019278.970 Neugeborene einem Neu-

Paediatr. Paedolog. 2021·56:59-66 https://doi.org/10.1007/s00608-021-00870-0

() Der/die Autor(en) 2021

\section{A. Eisenkölbl \\ Neue Therapiemöglichkeiten der spinalen Muskelatrophie. Brauchen wir ein Neugeborenen-Screening?}

\section{Zusammenfassung}

Seit einiger Zeit stehen für die Behandlung der spinalen Muskelatrophie (SMA) Medikamente mit unterschiedlichen Wirkmechanismen zur Verfügung, die den Verlauf der Erkrankung erheblich beeinflussen können. Unbehandelt ist diese neuromuskuläre Erkrankung immer progredient und führt bei der schwersten Verlaufsform SMA Typ 1 meist innerhalb von 24 Monaten zum Tod. Der genetische Defekt liegt auf dem Survival-motor-neuron1-Gen (SMN1-Gen). Dies führt zu einem Verlust von SMN1-Protein und damit zum Untergang von Motoneuronen. Bei allen Patienten liegt das SMN2-Gen, das nur etwa $10 \%$ funktionstüchtiges Protein bilden kann, in unterschiedlicher Kopienanzahl vor und beeinflusst den klinischen Schweregrad der Erkrankung, wobei fließende Übergänge zwischen den einzelnen Typen zu beobachten sind. Das erste für die SMA zugelassene Medikament ist Spinraza ${ }^{\circledR}$, ein AntisenseOligonukleotid, das intrathekal verabreicht wird, das mRNA-Splicing verändert und so zu einer vermehrten Produktion von SMN2-Protein führt. Das zweite zugelassene Medikament ist Zolgensma ${ }^{\circledR}$. Dabei handelt es sich um eine Genersatztherapie, bei der das SMN1-Gen mittels eines Virusvektors als Einmalinfusion in den Körper eingebracht wird, um dann funktionierendes SMN-Protein zu bilden. Kurz vor der Zulassung steht außerdem Risdiplam ${ }^{\circledR}$, dies ist ein sogenanntes "Small molecule" und setzt wie Spinraza ${ }^{\circledR}$ am SMN2-Gen an. Der Vorteil besteht in der Möglichkeit der oralen Einnahme. In allen Studien zu diesen Medikamenten wurde gezeigt, dass ein möglichst früher, am besten präsymptomatischer Beginn die besten Ergebnisse in den motorischen Scores für die Patienten erbrachte. Ein NeugeborenenScreening könnte die betroffenen Kinder noch vor Symptombeginn detektieren.

\section{Schlüsselwörter}

Behandlung · Phänotyp · Neuromuskuläre Erkrankung $\cdot$ Präsymptomatisch $\cdot$ Kinder

\section{New Therapeutic Options for Spinal Muscular Atrophy. Do We Need A Newborn Screening?}

\section{Abstract}

For some time now, drugs with different mechanisms of action have been available for the treatment of spinal muscular atrophy that can significantly influence the course of the disease. If left untreated, this neuromuscular disease is always progressive and the most severe form, SMA type 1, usually leads to death within 24 months. The genetic defect lies in the survival motor neuron 1 gene (SMN1 gene). This leads to a loss of SMN1 protein and thus to the death of motor neurons. In all patients, the SMN2 gene, which can only produce about $10 \%$ functional protein, is present in different numbers of copies and influences the clinical severity of the disease. Smooth transitions between the individual types are observed. The first drug approved for SMA is Spinraza ${ }^{\circledR}$, an antisense oligonucleotide that is administered intrathecally, changes mRNA splicing, and thus leads to increased production of SMN2 protein. The second approved drug is Zolgensma ${ }^{\circledR}$, a gene replacement therapy in which the SMN1 gene is introduced into the body as a single infusion using a virus vector, in order to produce functioning SMN protein Also about to be approved is Risdiplam ${ }^{\circledR}$, a socalled "small molecule" and, like Spinraza ${ }^{\circledR}$, it targets the SMN2 gene. The advantage is that it can be taken orally. In all studies on these drugs it could be shown that starting as early as possible, preferably presymptomatically, produced the best motor score results for the patients. Newborn screening could detect the affected children before symptoms start.

\section{Keywords}

Treatment · Phenotype $\cdot$ Neuromuscular disorder · Presymptomatic · Children 


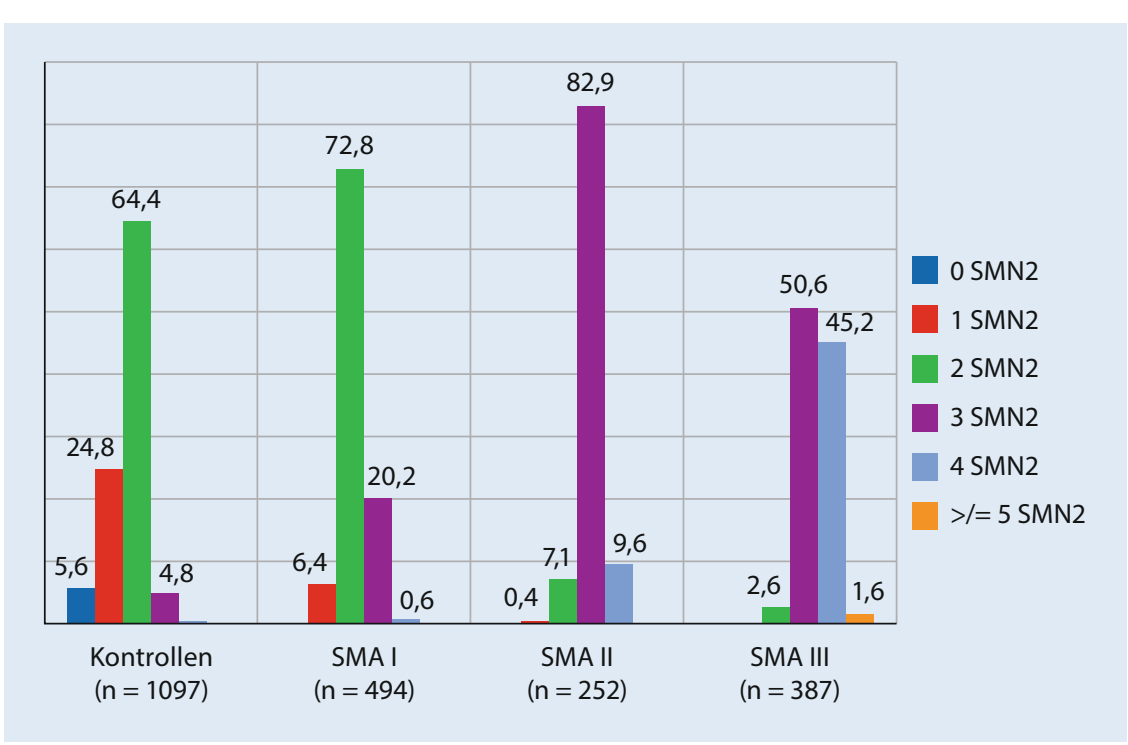

Abb. $2 \Delta$ Prozentuale Verteilung der SMN2-Genkopien bei Kontrollen und Patienten mit den Typen I-III der spinalen Muskelatrophie (SMA) (Modifiziert nach Rudnik-Schöneborn und Zerres [19])

geborenen-Screening zugeführt. Von diesen knapp 300.000 Neugeborenen wurde bei 38 Kindern eine homozygote Deletion auf dem SMN1-Gen detektiert. Die Inzidenz ergab somit einen Wert von $1 \mathrm{zu}$ 7350, was der Literatur entspricht.

Alle Familien wurden über den Befund informiert und ausführlich über die Erkrankung aufgeklärt. Alle Kinder wurden an Muskelzentren untersucht und je nach klinischer Symptomatik wurde entweder eine Therapie eingeleitet oder ein abwartendes Prozedere mit regelmäßigen Kontrollen vereinbart [14, 15].

Etwa $40 \%$ der Kinder hatten vier SMN2-Kopien und waren zum Zeitpunkt der Erstbegutachtung klinisch unauffällig. Kinder mit so einer hohen Kopienzahl stellen laut den Autoren die größte Herausforderung dar. Die Wahrscheinlichkeit für einen späten Krankheitsbeginn und einen milden Verlauf ist sehr groß, dennoch beschrieben die Autoren einen Patienten, der den klinischen Verlauf einer SMA 2 zeigte. Die Herausforderung besteht darin, diejenigen zu behandeln, die wahrscheinlich einen schwereren Verlauf haben werden, ohne die anderen überzubehandeln.

Weiterhin berichten die Kollegen aus Deutschland über Schwierigkeiten, (derzeit noch) asymptomatische Säuglinge über einen längeren Zeitraum in engmaschigen Kontrollen zu behalten. Die Gründe dafür wurden darin vermutet, dass die Eltern die Tragweite einer möglichen Erkrankung kognitiv nicht erfassen konnten bzw. dass die regelmäßigen Kontrollen den Eltern psychischen Stress verursacht haben.

Positiv wird hingegen die Reaktion der Familien berichtet, die in der erweiterten Familie bereits einen Patienten mit SMA haben und den Verlauf der Erkrankung kennen.

Und zusätzlich wurden über die Neugeborenen mehrere, klinisch auffällige, ältere Geschwister endlich einer Diagnose und somit einer Therapie zugeführt.

Aus medizinischer Sicht überwiegen daher die Vorteile eines NeugeborenenScreening für SMA, dennoch müssen Therapieentscheidungen gut überlegt und mit den betroffenen Familien besprochen werden.

\section{Zusätzliche Behandlungen - trotz neuer Therapien nicht vergessen}

In der Betreuung der betroffenen Patienten dürfen trotz der verfügbaren $\mathrm{Me}$ dikamente nicht die von Mercuri et al. sowie Finkel et al. zuletzt 2018 publizierten „Standards of Care“ vergessen werden [16, 17]. Diese Empfehlungen sind nach wie vor gültig und stellen die Ba- sis für eine optimale Patientenbetreuung dar. (• Abb. 4).

Damit dies gelingen kann, bedarf es eines multiprofessionellen Teams, wobei der Patient und seine Familie im Fokus stehen. Der Neuropädiater dient als Koordinator und somit als Schnittstelle zwischen den unterschiedlichen Professionen $[16,17]$.

\section{Neuromuskuläres bzw. orthopädisches Management und Rehabilitation}

Durch die Muskelschwäche und die eingeschränkte Mobilität ergibt sich eine deutliche Belastung des muskuloskelettalen Systems und damit ein hoher Bedarf an Hilfsmittelversorgung und physiotherapeutischer Unterstützung.

Im Vordergrund steht neben der funktionellen Therapie zur Kontrakturprophylaxe und Atemtherapie die Versorgung mit den optimalen Hilfsmitteln. Dabei handelt es sich um die verschiedensten Fortbewegungsmittel, wie zum Beispiel Reha-Buggy und Rollstuhl, aber auch um die Anpassung von Orthesen oder Korsetten.

Ein weiterer großer Bereich umfasst die operativen Versorgungsmöglichkeiten, wie zum Beispiel wirbelsäulenstabilisierende Operationen bei ausgeprägten Skoliosen oder Sehnenverlängerungen bei Kontrakturen wie zum Beispiel einer Spitzfußbildung.

\section{Ernährung und Knochengesundheit}

Gastroenterologische Probleme finden sich sehr häufig bei Patienten mit SMA. Meist geht es dabei um Fütterungsschwierigkeiten bzw. Schluckschwierigkeiten und damit einhergehend kommt es häufig zu einem schlechten Gedeihen. Neben verschiedensten Möglichkeiten der Nahrungsanreicherung bzw. Zugabe von hochkalorischen Nahrungsmitteln kann daher eine Anlage einer perkutanen endoskopischen Gastrostomiesonde notwendig werden. Aufgrund der Immobilität kann es zu gastrointestinalem Reflux, Obstipation und Osteoporose kommen. 


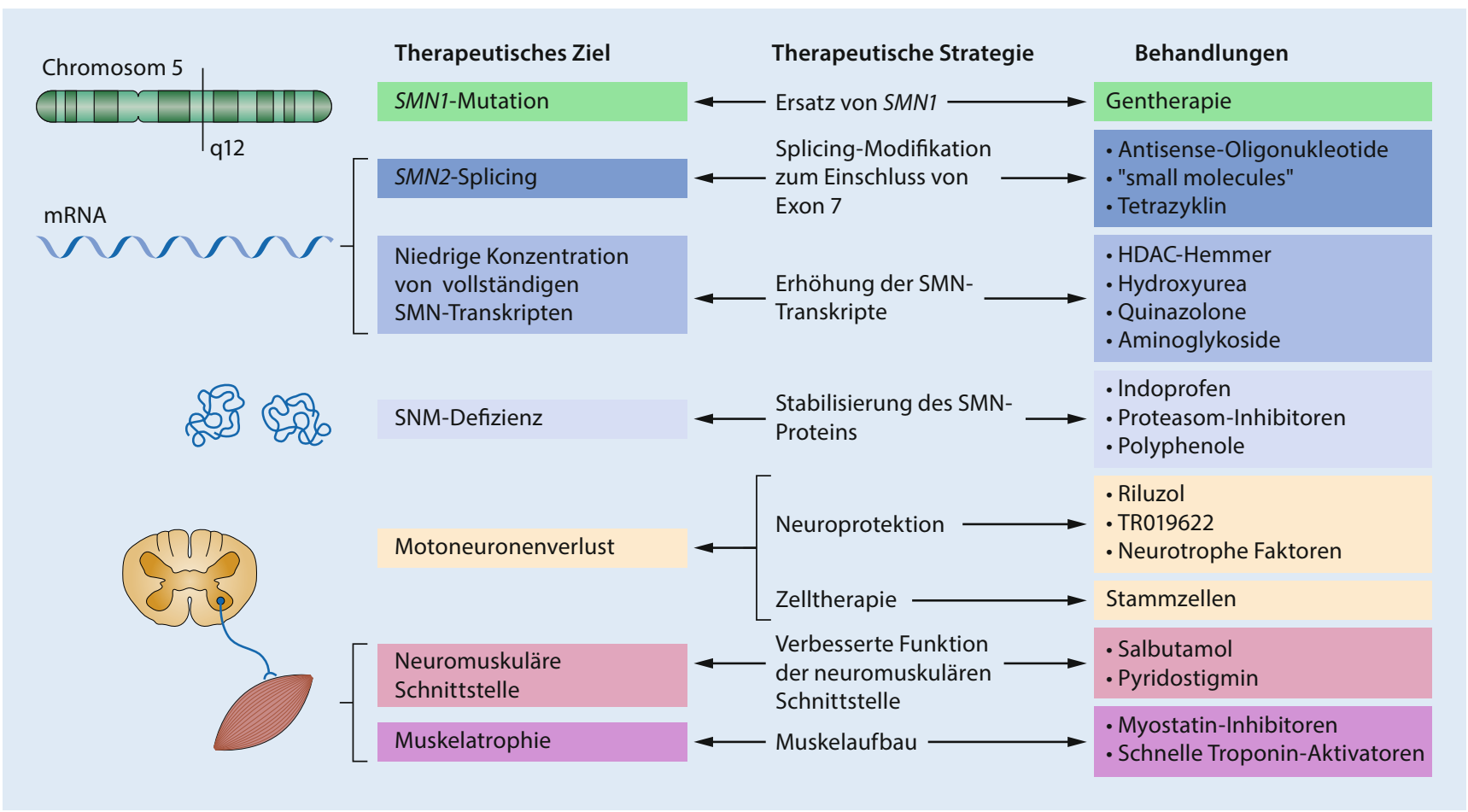

Abb. $3 \Delta$ Mögliche therapeutische Ziele, Strategien und Behandlungen für die spinale Muskelatrophie. HDAC Histondeacetylase [12]

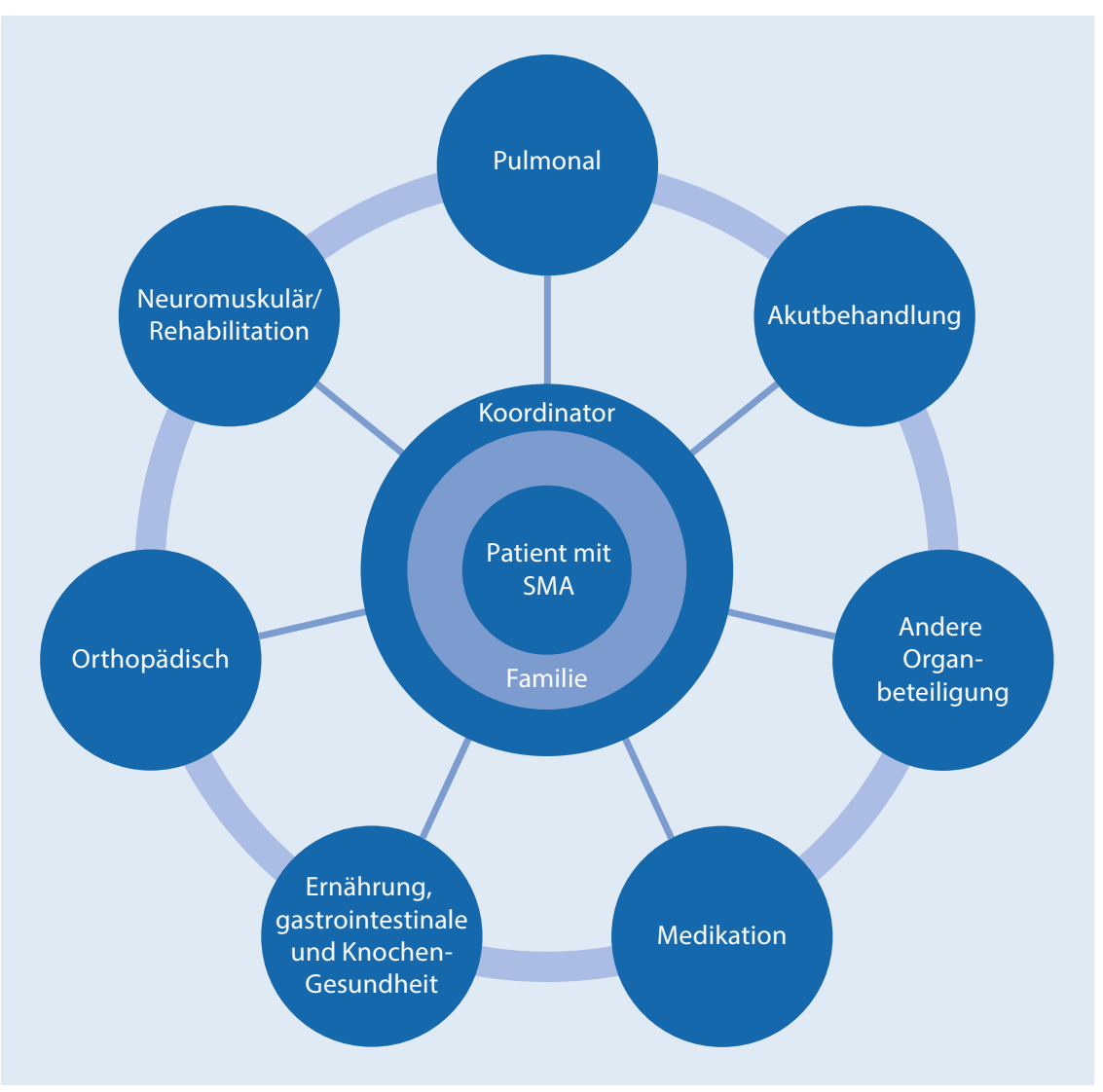

Die regelmäßige Kontrolle und gegebenenfalls Verabreichung von Vitamin D und Kalzium ist daher zur Vorbeugung von pathologischen Frakturen empfehlenswert, ebenso jährliche Knochendichtemessungen.

\section{Pulmonales Management}

Die vorrangige Todesursache sowohl bei Patienten mit SMA 1 als auch SMA 2 stellt das respiratorische Versagen dar.

Daher muss von Anfang an ein besonderes Augenmerk auf die pulmonale Situation gelegt werden, um Zeichen einer respiratorischen Insuffizienz frühzeitig zu erkennen. Die Patienten brauchen dann eine nichtinvasive Beatmung, später eventuell auch eine Beatmung über ein Tracheostoma.

Einen Großteil der Komplikationen der SMA machen sicherlich Atemwegsinfekte, insbesondere Pneumonien aus. Atemtherapie sollte daher immer ein Bestandteil der Therapie sein sowie die Durchführung von Impfungen gegen RSV, Influenza und Pneumokokken.

Abb. 4 \ "Standards of Care“ - Bedarf eines multiprofessionellen Teams [20] 


\section{Zusammenfassung}

- Neue Therapiemöglichkeiten haben deutliche Verbesserungen in den motorischen Scores sowie bei der Überlebensrate gezeigt.

- Je früher behandelt wurde, umso bessere Ergebnisse konnten erzielt werden (vor dem Verlust von Motoneuronen).

- Das Neugeborenen-Screening ermöglicht einen präsymptomatischen Therapiebeginn und damit den bestmöglichen Therapieerfolg.

- Neue Phänotypen bzw. fließende Übergänge zwischen den einzelnen SMA-Typen stellen eine Herausforderung im klinischen Alltag dar.

- Zusätzliche Behandlungsmöglichkeiten im Sinn der „Standards of Care" müssen die Basis der Therapie bleiben.

- Somit ist aus neuropädiatrischer Sicht die Aufnahme der SMA in das Neugeborenen-Screening zu empfehlen.

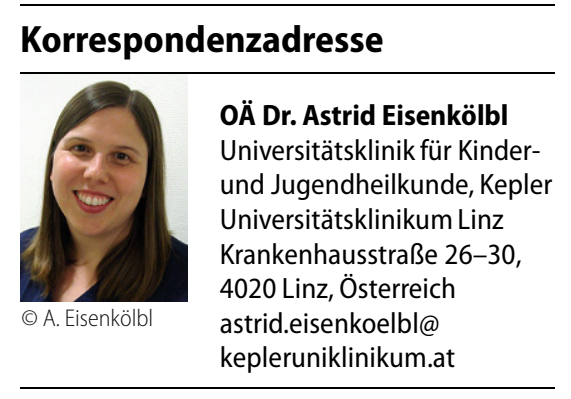

Hier steht eine Anzeige. Springer

Funding. Open access funding provided by Kepler Universitätsklinikum Linz.

\section{Einhaltung ethischer Richtlinien}

Interessenkonflikt. A. Eisenkölbl gibt an, dass sie Honorare für Beratungstätigkeiten und Vorträge für die Firmen Novartis, Roche und Sarepta erhalten hat.

Für diesen Beitrag wurden von den Autoren keine Studien an Menschen oder Tieren durchgeführt. Für die aufgeführten Studien gelten die jeweils dort angegebenen ethischen Richtlinien.

Open Access. Dieser Artikel wird unter der Creative Commons Namensnennung 4.0 International Lizenz veröffentlicht, welche die Nutzung, Vervielfältigung, Bearbeitung, Verbreitung und Wiedergabe in jeglichem Medium und Format erlaubt, sofern Sie den/die ursprünglichen Autor(en) und die Quelle ordnungsgemäß nennen, einen Link zur Creative Commons Lizenz 


\begin{tabular}{|c|c|c|c|}
\hline SMA-Typ & Alter bei Symptombeginn & Maximal erreichte motorische Funktion & Alter bei Tod \\
\hline 1 (Schwer) & Geburt bis 6 Monate & Unfähig zu sitzen & $<2$ Jahre \\
\hline 2 (Mittelschwer) & $<18$ Monate & Unfähig ohne Hilfe zu stehen oder zu gehen & $>2$ Jahre \\
\hline 3 (Mild) & 18 Monate bis 30 Jahre & Stehen und gehen & Erwachsen \\
\hline 4 (Sehr mild) & >30 Jahre & Stehen und gehen & Erwachsen \\
\hline
\end{tabular}

beifügen und angeben, ob Änderungen vorgenommen wurden.

Die in diesem Artikel enthaltenen Bilder und sonstiges Drittmaterial unterliegen ebenfalls der genannten Creative Commons Lizenz, sofern sich aus der Abbildungslegende nichts anderes ergibt. Sofern das betreffende Material nicht unter der genannten Creative Commons Lizenz steht und die betreffende Handlung nicht nach gesetzlichen Vorschriften erlaubt ist, ist für die oben aufgeführten Weiterverwendungen des Materials die Einwilligung des jeweiligen Rechteinhabers einzuholen.

Weitere Details zur Lizenz entnehmen Sie bitte der Lizenzinformation auf http://creativecommons.org/ licenses/by/4.0/deed.de.

\section{Literatur}

1. Kolb SJ, Kissel JT (2015) Spinal muscular atrophy. Neurol Clin 33:831-846. https://doi.org/10.1016/j. ncl.2015.07.004

2. Arnold ES, Fischbeck KH (2018) Spinal muscular atrophy. Handb Clin Neurol 148:591-601. https:// doi.org/10.1016/B978-0-444-64076-5.00038-7

3. D'Amico A, Mercuri E, Tiziano FD, Bertini E (2011) Spinal muscular atrophy. Orphanet J Rare Dis 6:71 https://doi.org/10.1186/1750-1172-6-71

4. Lefebvre S, Bürglen L, Reboullet $S$ et al (1995) Identification and characterization of a spinal muscular atrophy-determining gene. Cell 80:155-165. https://doi.org/10.1016/0092-8674(95)90460-3

5. Sieb JP, Schrank B (2009) Neuromuskuläre Erkrankungen. Kohlhammer, Stuttgart

6. Lorson CL, Hahnen E, Androphy EJ, Wirth B (1999) A single nucleotide in the SMN gene regulates splicing and is responsible for spinal muscular atrophy. Proc Natl Acad Sci USA 96:6307-6311. https://doi.org/10.1073/pnas.96.11.6307

7. Zerres K (1997) Spinal muscular atrophy — clinical and genetic correlations. Neuromuscul Disord 7:202-207. https://doi.org/10.1016/S09608966(97)00459-8

8. Zerres K, Rudnik-Schöneborn S (1995) Natural history in proximal spinal muscular atrophy. Clinical analysis of 445 patients and suggestions for a modification of existing classifications. Arch Neurol 52:518-523. https://doi.org/10.1001/ archneur.1995.00540290108025

9. Munsat TL, Davies KE (1992) International SMA consortium meeting (26-28 June 1992, Bonn, Germany). Neuromuscul Disord 2:423-428. https://doi.org/10.1016/S0960-8966(06)80015-5

10. Finkel RS, Chiriboga CA, Vajsar J et al (2016) Treatment of infantile-onset spinal muscular atrophy with nusinersen: a phase 2, open-label dose-escalation study. Lancet 388:3017-3026. https://doi.org/10.1016/S0140-6736(16)31408-8

11. Finkel RS, Mercuri E, Darras BT et al (2017) Nusinersen versus sham control in infantile-onset spinal muscular atrophy. N Engl J Med 377:1723-1732. https://doi.org/10.1056/NEJMoa1702752

12. Mercuri E, Pera MC, Scoto $M$, Finkel R, Muntoni $F$ (2020) Spinal muscular atrophy_-insights and challenges in the treatment era. Nat Rev Neurol. https://doi.org/10.1038/s41582-020-00413-4

13. Mendell JR, Al-Zaidy S, Shell R et al (2017) Single-dose gene-replacement therapy for spinal muscular atrophy. N Engl J Med 377:1713-1722. https://doi.org/10.1056/NEJMoa1706198

14. Müller-Felber W, Vill K, Schwartz O et al (2020) Infants diagnosed with spinal muscular atrophy and 4 SMN2 copies through newborn screening-opportunity or burden? J Neuromuscul Dis 7:109-117. https://doi.org/10.3233/JND-200475

15. Vill K, Kölbel H, Schwartz O et al (2019) One year of newborn screening for SMA - results of a German pilot project. J Neuromuscul Dis 6:503-515. https://doi.org/10.3233/JND-190428

16. Finkel RS, Mercuri E, Meyer $\mathrm{OH}$ et al (2018) Diagnosis and management of spinal muscular atrophy: part 2: pulmonary and acute care; medications, supplements and immunizations; other organ systems; and ethics. Neuromuscul Disord. https://doi.org/10.1016/j.nmd.2017.11. 004

17. Mercuri E, Finkel RS, Muntoni F et al (2018) Diagnosis and management of spinal muscular atrophy: part 1: recommendations for diagnosis, rehabilitation, orthopedic and nutritional care. Neuromuscul Disord. https://doi.org/10.1016/j. nmd.2017.11.005

18. Chen T-H (2020) New and developing therapies in spinal muscular atrophy: from genotype to phenotype to treatment and where do we stand? Int J Mol Sci 21(9):3297. https://doi.org/10.3390/ ijms21093297

19. Rudnik-Schöneborn S, Zerres K (2017) Spinale Muskelatrophien. medgen 29:21-34

20. Mercuri E et al (2018) Diagnosis and management of spinal muscular atrophy: part 1 recommendations for diagnosis, rehabilitation, orthopedic and nutritional care. Neuromuscul Disord 28(2):103-115

Hinweis des Verlags. Der Verlag bleibt in Hinblick auf geografische Zuordnungen und Gebietsbezeichnungen in veröffentlichten Karten und Institutsadressen neutral. 